\title{
Buckling Analysis of a Rotationally Restrained Single Walled Carbon Nanotube
}

\author{
M.Ö. YAYLI* \\ Bilecik Şeyh Edebali University, Faculty of Engineering, Department of Civil Engineering, \\ 11210 Gülümbe Kampüsü, Bilecik, Turkey
}

(Received June 4, 2014; in final form January 22, 2015)

\begin{abstract}
This paper presents an analytical formulation of nonlocal elasticity theory for the buckling analysis of simply supported carbon nanotubes with rotational springs at both ends. The lateral displacement function is represented by a Fourier sine series expansion. Stoke's transformation is applied to construct the coefficient matrix of the corresponding systems of linear equations. This matrix gives more flexibility in boundary conditions. The accuracy of proposed method is validated for three well-known boundary conditions available in the literature. A very good agreement has been obtained. The present method permits to have more efficient stability matrix for calculating the buckling loads of carbon nanotubes with any desired boundary conditions.
\end{abstract}

DOI: $10.12693 /$ APhysPolA.127.678

PACS: 03.65.Db, 02.30.Nw, 02.30.Hq, 61.46.+w

\section{Introduction}

Carbon nanotubes have many applications in engineering practice because of excellent mechanical properties, such as chemical and thermal stability, hollow geometry, flexibility, large Young's modulus, yield strength, and conductivity properties [1]. Thermal and electrical properties of carbon nanotube based materials are studied by Yuca et al. [2]. Gas sensing properties of carbon nanotubes (CNTs) are explored by Sumer et al. [3]. From the literature review, there are a lot of studies available related to mechanical properties of carbon nanotubes.

Classical elasticity models lack the accountability of the effects arising from the small scale. Thus, application of classical elasticity theories may be questionable in the analysis of carbon nanotubes. Due to the presence of small size effects at the micro/nanoscale, small sizedependent elasticity models such as the nonlocal elasticity theory [4-6], modified couple stress theory $[7,8]$, the strain gradient theory [9] are used. Among these models, nonlocal elasticity approach is widely used due to its computational efficiency. Unlike the classical elasticity theory, the nonlocal elasticity theory assumes that stress at a point depends not only on the strain at that point but also on strains at all other points of the body. Nonlocal elasticity theory takes into account the forces between atoms.

Static analysis of nanostructures has been presented by Wang and Liew [10] using the nonlocal Timoshenko and Euler-Bernoulli beam theories. Kiani [11-14] have studied vibration, buckling, and wave propagation in single walled carbon nanotubes (SWCNTs) and their ensembles in the context of the nonlocal continuum theory of Eringen [4]. Peddieson et al. [15] have proposed nonlocal Euler-Bernoulli model for static analysis of nanobeams.

*e-mail: mozgur.yayli@bilecik.edu.tr
Elastic buckling behavior of single walled carbon nanotubes has also attracted many investigators. These structures are assumed as a beam model for calculating the buckling loads. Axial buckling analysis of single-walled carbon nanotubes in thermal environments has been considered by Ansari et al. [16]. There have been many studies dedicated to the buckling analysis of carbon nanotubes using nonlocal elasticity [17-22]. Numerical results in these studies are presented to bring out the nonlocal effects on buckling loads of carbon nanotubes.

It appears that most of the previous investigations on the stability analysis carbon nanotubes are mostly limited to classical rigid boundary conditions. However, a variety of restrained boundary conditions are usually encountered in many micro/nanostructure applications. Existence of rotational springs within these types of structures leads to changes in critical buckling loads of the nanotubes. In nonlocal elasticity, obtaining exact results for the buckling behavior of carbon nanotubes with end springs are almost impossible in most of the cases. Therefore, it is important to develop an analytical method which is capable of dealing with carbon nanotubes with restrained boundary conditions. In this study, a compact analytical method is introduced for the stability analysis of a simply supported single walled carbon nanotube with rotational springs at both ends. The displacement field of the carbon nanotube under compression is represented by a Fourier sine series. The stability determinant is constructed by applying Stoke's transformation to the boundary conditions. This determinant can also be used to determine the buckling loads of a carbon nanotube with rigid boundary conditions such as clamped-clamped, clamped-simply supported. The reliability of the presented analytical method are adequately validated through numerical examples. Analytical solutions presented herein should serve as references for buckling analysis of carbon nanotubes with any desired boundary conditions. 


\section{The analytical formulation for buckling}

\subsection{A brief review of nonlocal elasticity}

The basic partial integral stress equation for a linear, isotropic and homogeneous nonlocal elastic body neglecting the body force is expressed as [4]:

$$
\sigma_{i j}(z)=\int_{V} \ell\left(\left|z-z^{\prime}\right|, \beta\right) t_{i j} d V\left(z^{\prime}\right), \quad \forall z \in V .
$$

The terms $t_{i j}$ and $\sigma_{i j}$ are the classical stress and nonlocal stress tensors, respectively. The three-dimensional volume integral is over the region $V$ occupied by the body. The function $\ell\left(\left|z-z^{\prime}\right|, \beta\right)$ is the nonlocal modulus. $\beta$ is a material constant. The term $\left|z-z^{\prime}\right|$ represents the distance in the Euclidean form. The partial integral stress equation in (1) is generally difficult to solve analytically. Therefore, a differential form is often used as follows:

$$
\left(1-\left(e_{0} a\right)^{2} \nabla^{2}\right) \sigma_{i j}^{\mathrm{NL}}=\sigma_{i j}^{\mathrm{L}},
$$

where $\left(e_{0} a\right)^{2}$ is the nonlocal parameter, $\sigma_{i j}^{\mathrm{L}}$ and $\sigma_{i j}^{\mathrm{NL}}$ are local and nonlocal stresses. For a nonlocal EulerBernoulli beam, constitutive relations can be expressed in a differential form as [6]:

$$
\sigma_{z z}-\left(e_{0} a\right)^{2} \frac{\partial^{2} \sigma_{z z}}{\partial z^{2}}=E \epsilon_{z z},
$$

$E, \sigma_{z z}$ and $\epsilon_{z z}$ denote the Young modulus, stress, and the strain in the nonlocal beam. With the help of (3) the constitutive relation in nonlocal elasticity can be written as follows:

$$
M-\left(e_{0} a\right)^{2} \frac{\mathrm{d}^{2} M}{\mathrm{~d} z^{2}}=-E I \frac{\mathrm{d}^{2} w}{\mathrm{~d} z^{2}},
$$

where $M$ denotes the bending moment. Then, governing differential equation for the buckling is given by [1]:

$$
E I \frac{\mathrm{d}^{4} w}{\mathrm{~d} z^{2}}+P \frac{\mathrm{d}^{2}}{\mathrm{~d} z^{2}}\left[w-\left(e_{0} a\right)^{2} \frac{\mathrm{d}^{2} w}{\mathrm{~d} z^{2}}\right]=0,
$$

Here $E I$ is the flexural rigidity, $P$ is the compression load. In order to solve Eq. (5) investigators preferred differrential transform method (DTM) [23, 24]. However, with differrential transform method the above governing differential equation cannot be related to the restrained boundary conditions. Therefore, another transformation should be employed to solve this nonclassical boundary value problem.

\subsection{Lateral displacement function for rotational restraints}

To investigate the significance of using rotational restraints on the buckling loads of carbon nanotubes, a simply supported single walled carbon nanotube with rotational restraints is considered. The rotational restraints at the ends of carbon nanotube are modelled using rotational springs (see Fig. 1).

The lateral displacement function $w(z)$ under axial load $(P)$ is described in the following separate form:

$$
w(z)=\phi(z)=\left[\begin{array}{cc}
\phi_{0} & z=0, \\
\phi_{L} & z=L, \\
\sum_{n=1}^{\infty} \vartheta_{n} \sin \left(\frac{n \pi z}{L}\right) & 0<z<L .
\end{array}\right]
$$

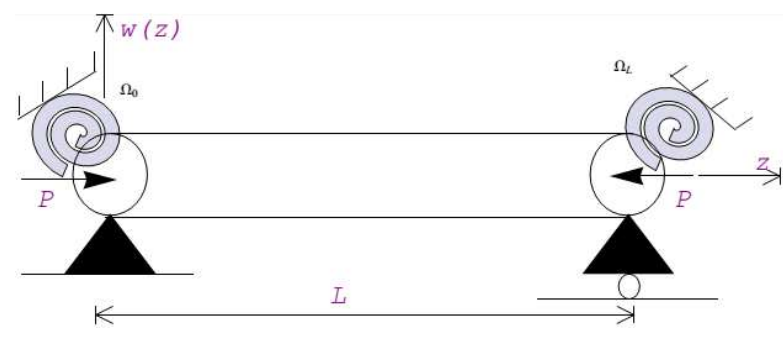

Fig. 1. A simply supported carbon nanotube with rotational springs at both ends.

It should be noted that regardless of supporting conditions, the lateral displacement function can be expressed as any Fourier series expansion. Therefore, the Fourier cosine series may also be used to represent the lateral displacement function.

\subsection{Stoke's transformation}

The Fourier sine coefficients in (6) are obtained by considering the following equation:

$$
\vartheta_{n}=\frac{2}{L} \int_{0}^{L} \phi(z) \sin \left(\frac{n \pi z}{L}\right) \mathrm{d} x,
$$

Termwise differentiation of Eq. (6) yields

$$
\phi^{\prime}(z)=\sum_{n=1}^{\infty} \frac{n \pi}{L} \vartheta_{n} \cos \left(\frac{n \pi z}{L}\right),
$$

Above equation can be represented by a Fourier cosine series,

$$
\phi^{\prime}(z)=\frac{C_{0}}{L}+\sum_{n=1}^{\infty} C_{n} \cos \left(\frac{n \pi z}{L}\right),
$$

The coefficients are given by

$$
\begin{aligned}
& C_{0}=\frac{2}{L} \int_{0}^{L} \phi^{\prime}(z) \mathrm{d} z=\frac{2}{L}[\phi(L)-\phi(0)], \\
& C_{n}=\frac{2}{L} \int_{0}^{L} \phi^{\prime}(z) \cos \left(\frac{n \pi z}{L}\right) \mathrm{d} z, \quad n=1,2 \ldots
\end{aligned}
$$

Integration by parts

$$
\begin{aligned}
C_{n} & =\frac{2}{L}\left[\phi(z) \cos \left(\frac{n \pi z}{L}\right)\right]_{0}^{L} \\
+ & \frac{2}{L}\left[\frac{n \pi}{L} \int_{0}^{L} \phi(z) \sin \left(\frac{n \pi z}{L}\right) \mathrm{d} z\right], \\
C_{n} & =\frac{2}{L}\left[(-1)^{n} \phi(L)-\phi(0)\right]+\frac{n \pi}{L} \vartheta_{n} .
\end{aligned}
$$

The higher order derivatives can be achieved with the use of the similar procedure as follows:

$$
\begin{aligned}
& \frac{\mathrm{d} w(z)}{\mathrm{d} z}=\frac{\phi_{L}-\phi_{0}}{L} \\
& \quad+\sum_{n=1}^{\infty} \cos \left(\alpha_{n} z\right)\left(\frac{2\left((-1)^{n} \phi_{L}-\phi_{0}\right)}{L}+\alpha_{n} \vartheta_{n}\right), \\
& \frac{\mathrm{d}^{2} w(z)}{\mathrm{d} z^{2}}=-\sum_{n=1}^{\infty} \alpha_{n} \sin \left(\alpha_{n} z\right) \\
& \quad \times\left(\frac{2\left((-1)^{n} \phi_{L}-\phi_{0}\right)}{L}+\alpha_{n} \vartheta_{n}\right)
\end{aligned}
$$




$$
\begin{aligned}
& \frac{\mathrm{d}^{3} w(z)}{\mathrm{d} z^{3}}=\frac{\phi_{L}^{\prime \prime}-\phi_{0}^{\prime \prime}}{L}+\sum_{n=1}^{\infty} \cos \left(\alpha_{n} z\right)\left(\frac{2\left((-1)^{n} \phi_{L}^{\prime \prime}-\phi_{0}^{\prime \prime}\right)}{L}\right. \\
&\left.-\alpha_{n}^{2}\left(\frac{2\left((-1)^{n} \phi_{L}-\phi_{0}\right)}{L}+\alpha_{n} \vartheta_{n}\right)\right), \\
& \frac{\mathrm{d}^{4} w(z)}{\mathrm{d} z^{4}}=-\sum_{n=1}^{\infty} \alpha_{n} \sin \left(\alpha_{n} z\right)\left(\frac{2\left((-1)^{n} \phi_{L}^{\prime \prime}-\phi_{0}^{\prime \prime}\right)}{L}\right. \\
&\left.-\alpha_{n}^{2}\left(\frac{2\left((-1)^{n} \phi_{L}-\phi_{0}\right)}{L}+\alpha_{n} \vartheta_{n}\right)\right),
\end{aligned}
$$

where

$$
\alpha_{n}=\frac{n \pi}{L} .
$$

Substituting (6), (15) and (17) into (5) leads to the following equation:

$$
\begin{aligned}
& \frac{\alpha_{n} \sin \left(\alpha_{n} z\right)}{L} \times\left(L \alpha_{n}^{3} \vartheta_{n} \delta_{P}-2 \alpha_{n}^{2} \delta_{P}\left(\phi_{0}-(-1)^{n} \phi_{L}\right)\right. \\
& +2\left(\delta_{P}\left(\phi_{0}^{\prime \prime}-(-1)^{n} \phi_{L}^{\prime \prime}\right)-(-1)^{n} P \phi_{L}+P \phi_{0}\right) \\
& \left.-L P \alpha_{n} \vartheta_{n}\right)=0,
\end{aligned}
$$

where

$$
\delta_{P}=E I-\left(e_{0} a\right)^{2} P .
$$

The coefficient $\vartheta_{n}$ can be written as follows:

$$
\begin{aligned}
\vartheta_{n} & =\frac{2\left((-1)^{n} \phi_{L}\left(\alpha_{n}^{2} \delta_{P}-P\right)\right)}{L \alpha_{n}\left(-\alpha_{n}^{2} \delta_{P}-P\right)} \\
& +\frac{2\left(\delta_{P}\left(\phi_{0}^{\prime \prime}-(-1)^{n} \phi_{L}^{\prime \prime}\right)-\phi_{0}\left(\alpha_{n}^{2} \delta_{P}-P\right)\right)}{L \alpha_{n}\left(-\alpha_{n}^{2} \delta_{P}-P\right)}
\end{aligned}
$$

and the lateral displacement function can be interpreted as a sum of the Fourier components

$$
\begin{aligned}
& \phi(z)=\sum_{n=1}^{\infty} \frac{2\left((-1)^{n} \phi_{L}\left(\alpha_{n}^{2} \delta_{P}-P\right)\right)}{L \alpha_{n}\left(-\alpha_{n}^{2} \delta_{P}-P\right)} \\
& +\frac{2\left(\delta_{P}\left(\phi_{0}^{\prime \prime}-(-1)^{n} \phi_{L}^{\prime \prime}\right)-\phi_{0}\left(\alpha_{n}^{2} \delta_{P}-P\right)\right)}{L \alpha_{n}\left(-\alpha_{n}^{2} \delta_{P}-P\right)} \\
& \quad \times \sin \left(\alpha_{n} z\right),
\end{aligned}
$$

when the nonlocal parameter $\left(e_{0} a^{2}\right)$ taken as zero, above formulation turns to be useful for local elasticity.

\section{Legitimized stability expressions}

Consider a simply supported single walled carbon nanotube of length $L$ which is assumed to be elastically restrained by means of rotational springs (see Fig. 1). Then the prescribed boundary conditions are

$$
\begin{aligned}
& \phi_{0}=0, \quad z=0, \\
& \phi_{L}=0, \quad z=L, \\
& \Omega_{0} \frac{\mathrm{d} \phi}{\mathrm{d} x}=E I \frac{\mathrm{d}^{2} \phi(z)}{\mathrm{d} z^{2}}, \quad x=0, \\
& \Omega_{\mathrm{L}} \frac{\mathrm{d} \phi}{\mathrm{d} x}=-E I \frac{\mathrm{d}^{2} \phi(z)}{\mathrm{d} z^{2}}, \quad x=L,
\end{aligned}
$$

where $\Omega_{0}$ and $\Omega_{\mathrm{L}}$ are the rotational spring constants. Substituting (14) and (16) into above boundary conditions leads to two homogeneous equations

$$
\begin{aligned}
& \left(-1+\Omega_{0} \sum_{n=1}^{\infty} \frac{2 L(E I-\mu P)}{E I\left(\pi^{2} n^{2}(\mu P-E I)+L^{2} P\right)}\right) \phi_{0}^{\prime \prime} \\
& -\left(\Omega_{0} \sum_{n=1}^{\infty} \frac{2 L(-1)^{n}(E I-\mu P)}{E I\left(\pi^{2} n^{2}(\mu P-E I)+L^{2} P\right)}\right) \phi_{L}^{\prime \prime}=0, \\
& -\left(\Omega_{L} \sum_{n=1}^{\infty} \frac{2 L(-1)^{n}(E I-\mu P)}{E I\left(\pi^{2} n^{2}(\mu P-E I)+L^{2} P\right)}\right) \phi_{0}^{\prime \prime} \\
& +\left(-1+\Omega_{L} \sum_{n=1}^{\infty} \frac{2 L(E I-\mu P)}{E I\left(\pi^{2} n^{2}(\mu P-E I)+L^{2} P\right)}\right) \phi_{L}^{\prime \prime}=0,
\end{aligned}
$$

where

$$
\mu=\left(e_{0} a\right)^{2}
$$

and the following system of linear algebraic equations are achieved in a matrix form to be solved for the constants $\left(\phi_{0}^{\prime \prime}\right.$ and $\left.\phi_{L}^{\prime \prime}\right)$ :

$$
\left[\begin{array}{ll}
\eta_{11} & \eta_{12} \\
\eta_{21} & \eta_{22}
\end{array}\right]\left[\begin{array}{l}
\phi_{0}^{\prime \prime} \\
\phi_{L}^{\prime \prime}
\end{array}\right]=0 .
$$

The characteristic equation is derived by setting the determinant of the above coefficient matrix equal to zero. Then, the resultant determinant is written as

$$
\left|\eta_{i j}\right|=0 ; \quad(i, j=1,2)
$$

where

$$
\begin{aligned}
& \eta_{11}=-1+\Omega_{0} \sum_{n=1}^{\infty} \frac{2 L(E I-\mu P)}{E I\left(\pi^{2} n^{2}(\mu P-E I)+L^{2} P\right)} \\
& \eta_{12}=-\Omega_{0} \sum_{n=1}^{\infty} \frac{2 L(-1)^{n}(E I-\mu P)}{E I\left(\pi^{2} n^{2}(\mu P-E I)+L^{2} P\right)} \\
& \eta_{21}=-\Omega_{L} \sum_{n=1}^{\infty} \frac{2 L(-1)^{n}(E I-\mu P)}{E I\left(\pi^{2} n^{2}(\mu P-E I)+L^{2} P\right)}, \\
& \eta_{22}=-1+\Omega_{L} \sum_{n=1}^{\infty} \frac{2 L(E I-\mu P)}{E I\left(\pi^{2} n^{2}(\mu P-E I)+L^{2} P\right)} .
\end{aligned}
$$

The smallest eigenvalue corresponding to the critical buckling load can be determined by using (31).

\section{Numerical results}

In this section, a simply supported carbon nanotube with rotational springs at both ends is analyzed by the presented method. Firstly, to verify the accuracy of the presented method, a comparison of the critical buckling loads in classical elasticity with the same results available in the literature for the carbon nanotubes with classical supporting conditions is carried out.

\subsection{Verification of the presented method}

In this subsection, for the purpose of verification, the present formulation is used to find the critical buckling load of a carbon nanotube where the material scale parameter $(\mu)$ is taken as zero and compare the results with those available in the literature.

The material properties are taken as $E=1 \mathrm{TPa}$, diameter of nanotube $d=1 \mathrm{~nm}$. It should be noted that by letting ( $\left.\Omega_{0}=\Omega_{L}=0\right)$, Eq. (31) will automatically degenerate into the simply supported carbon nanotube. 
Therefore, $\Omega_{0}=\Omega_{L}=1 \times 10^{-9} \mathrm{nN} \mathrm{nm} /(\mathrm{nm} \mathrm{rad})$ values are utilized in the present verification.

In the second validation, spring parameters are taken as $\left.\left(\Omega_{0}=\Omega_{L}=1 \times 10^{+9} \mathrm{nN} \mathrm{nm/(nm} \mathrm{rad}\right)\right)$ for the carbon nanotube with clamped-clamped ends and results are tabulated in Table I.

TABLE I

Verification of the proposed method for a carbon nanotube with clamped-clamped ends.

\begin{tabular}{c|c|c|c}
\hline \hline \multirow{2}{*}{ Length [nm] } & \multicolumn{3}{|c}{$P[\mathrm{nN}]$} \\
\cline { 2 - 4 } & $P_{\text {exact }}[24]$ & $P_{\text {DTM }}[25]$ & $P_{\text {FSE }}$ \\
\hline 10 & 19.379 & 19.379 & 19.379 \\
12 & 13.458 & 13.458 & 13.458 \\
14 & 9.877 & 9.877 & 9.877 \\
16 & 7.4699 & 7.4699 & 7.4699 \\
18 & 5.9811 & 5.9811 & 5.9811 \\
20 & 4.8447 & 4.8447 & 4.8447
\end{tabular}

TABLE II

Verification of the proposed method for a simply supported carbon nanotube.

\begin{tabular}{c|c|c|c}
\hline \hline Length [nm] & \multicolumn{3}{|c}{$P[\mathrm{nN}]$} \\
\cline { 2 - 4 } & $P_{\text {exact }}[24]$ & $P_{\text {DTM }}[25]$ & $P_{\text {FSE }}$ \\
\hline 10 & 4.8447 & 4.8447 & 4.8447 \\
12 & 3.3644 & 3.3644 & 3.3644 \\
14 & 2.4718 & 2.4718 & 2.4718 \\
16 & 1.8925 & 1.8925 & 1.8925 \\
18 & 1.4953 & 1.4953 & 1.4953 \\
20 & 1.2112 & 1.2112 & 1.2112
\end{tabular}

By letting $\left(\Omega_{0}=1 \times 10^{+9}\right.$ and $\Omega_{L}=1 \times$ $\left.10^{-9} \mathrm{nN} \mathrm{nm/(nm} \mathrm{rad)}\right)$, Eq. (31) could be used for the clamped-pinned carbon nanotube.

TABLE III

Verification of the proposed method for a clamped-pinned carbon nanotube.

\begin{tabular}{c|c|c|c}
\hline \hline Length [nm] & \multicolumn{3}{|c}{$P[\mathrm{nN}]$} \\
\cline { 2 - 4 } & $P_{\text {exact }}[24]$ & $P_{\text {DTM }}[25]$ & $P_{\text {FSE }}$ \\
\hline 10 & 9.887 & 9.887 & 9.887 \\
12 & 6.886 & 6.886 & 6.886 \\
14 & 5.044 & 5.044 & 5.044 \\
16 & 3.8621 & 3.8621 & 3.8621 \\
18 & 3.0516 & 3.0516 & 3.0516 \\
20 & 2.4718 & 2.4718 & 2.4718
\end{tabular}

In Tables I-III, the critical buckling loads for the carbon nanotubes are given and compared with exact results and also with the differrential transform method (DTM) results. All critical buckling loads are determined by using the first 200 terms of the Fourier series expansion (FSE). It is seen that the presented results and the exact results are in good agreement. It can be concluded that the present results seem to be more acceptable.

A convergence check study is also provided to show how the critical axial load of the rotationally restrained carbon nanotube would converge by an increase of the number of series' terms. For this aim, the percentage difference $(P D)$ is defined as follows:

$$
P D=\frac{P_{\mathrm{FSE}}-P_{\text {exact }}}{P_{\text {exact }}} \times 100 \text {. }
$$

The nonlocal parameter is taken as zero, length of nanotube $L=20 \mathrm{~nm}$. It is clear from Eq. (6) that Fourier sine series satisfies only the natural boundary conditions of a nanotube whose boundaries are simply supported. Thus, a convergence study is carried out for clamped-clamped and clamped-pinned boundary conditions. In Figure 2 the variation of percentage difference $(\mathrm{PD})$ with the number of terms used in the Fourier series is depicted. It is seen that 80 terms of Fourier series can be satisfactory to obtain the buckling load with accuracy lower than $1 \%$.

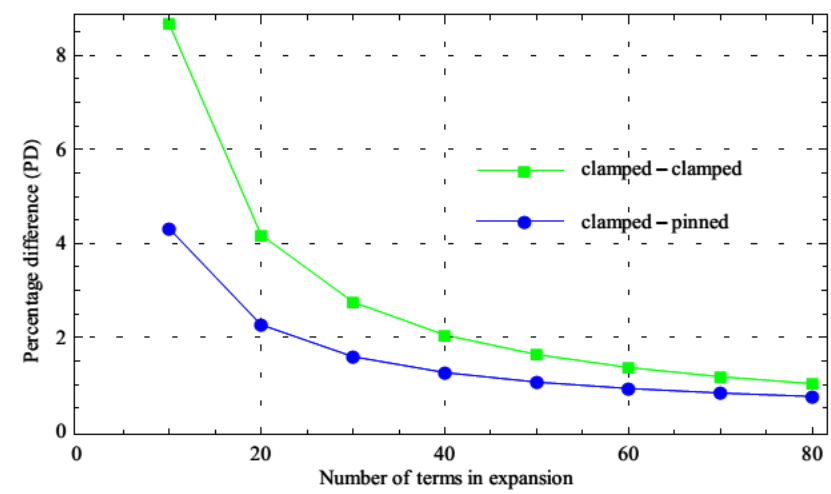

Fig. 2. Graph of percentage difference (PD) against number of terms in expansion.

\subsection{Results and discussion}

In this section, various numerical examples are solved and discussed to asses the accuracy of the presented method in predicting the stability behaviour of carbon nanotubes. The carbon nanotube has the following material properties: $E=1 \mathrm{TPa}$. The diameter of nanotube is taken as $1 \mathrm{~nm}$. In general, the nonlocal parameter is $(\mu)<2 \mathrm{~nm}^{2}$ for a single wall carbon nanotube [26]. Therefore, in this paper, the nonlocal parameter is taken as $(\mu=0,0.3,0.5,1.0) \mathrm{nm}^{2}$ to investigate nonlocal effects on the buckling responses of carbon nanotube.

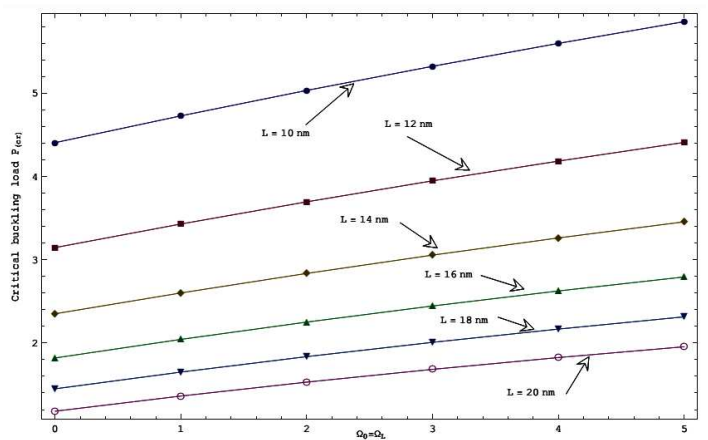

Fig. 3. Variation of the critical buckling loads $\left(P_{\mathrm{cr}}\right)$ for $\left(\mu=1.0 \mathrm{~nm}^{2}\right)$. 
Variation of the critical buckling loads $\left(P_{\mathrm{cr}}\right)$ for $\left(\mu=1.0 \mathrm{~nm}^{2}\right)$.

\begin{tabular}{c|c|c|c|c|c|c|c|c}
\hline \hline \multirow{2}{*}{$\mathrm{L}[\mathrm{nm}]$} & \multicolumn{2}{|c|}{$\mu=1.0 \mathrm{~nm}^{2}$} & \multicolumn{5}{c}{ Present $\mu=1.0 \mathrm{~nm}^{2}$} \\
\cline { 2 - 8 } & Ref. [24] & Ref. [25] & $\Omega_{0}=\Omega_{L}=0$ & $\Omega_{0}=\Omega_{L}=1$ & $\Omega_{0}=\Omega_{L}=2$ & $\Omega_{0}=\Omega_{L}=3$ & $\Omega_{0}=\Omega_{L}=4$ & $\Omega_{0}=\Omega_{L}=5$ \\
\hline 10 & 4.4095 & 4.4095 & 4.40953 & 4.73207 & 5.03774 & 5.32763 & 5.60275 & 5.86404 \\
12 & 3.1486 & 3.1486 & 3.14860 & 3.43195 & 3.69893 & 3.95068 & 4.18825 & 4.41260 \\
14 & 2.3533 & 2.3533 & 2.35330 & 2.60388 & 2.83850 & 3.05836 & 3.26456 & 3.45813 \\
16 & 1.8222 & 1.8222 & 1.82222 & 2.04575 & 2.25367 & 2.44724 & 2.62762 & 2.79587 \\
18 & 1.4511 & 1.4511 & 1.45108 & 1.65223 & 1.83804 & 2.00987 & 2.16892 & 2.31632 \\
20 & 1.1820 & 1.1820 & 1.18202 & 1.36446 & 1.53182 & 1.68551 & 1.82681 & 1.95691
\end{tabular}

TABLE V

Effect of nonlocal parameter on the buckling load of carbon nanotube.

\begin{tabular}{c|c|c|c|c|c|c|c|c}
\hline \hline \multirow{2}{*}{$\mathrm{L}[\mathrm{nm}]$} & \multicolumn{2}{|c|}{$\mu=0.0 \mathrm{~nm}^{2}$} & \multicolumn{2}{c|}{$\mu=0.3 \mathrm{~nm}^{2}$} & \multicolumn{2}{c|}{$\mu=0.5 \mathrm{~nm}^{2}$} & \multicolumn{2}{c}{$\mu=1.0 \mathrm{~nm}^{2}$} \\
\cline { 2 - 8 } & $\Omega_{0}=\Omega_{L}=1$ & $\Omega_{0}=\Omega_{L}=5$ & $\Omega_{0}=\Omega_{L}=1$ & $\Omega_{0}=\Omega_{L}=5$ & $\Omega_{0}=\Omega_{L}=1$ & $\Omega_{0}=\Omega_{L}=5$ & $\Omega_{0}=\Omega_{L}=1$ & $\Omega_{0}=\Omega_{L}=5$ \\
\hline 10 & 5.2369 & 6.6596 & 5.0745 & 6.3991 & 4.9717 & 6.2365 & 4.7321 & 5.8640 \\
12 & 3.6899 & 4.8484 & 3.6085 & 4.7089 & 3.5562 & 4.6203 & 3.4319 & 4.4126 \\
14 & 2.7497 & 3.7202 & 2.7043 & 3.6375 & 2.6748 & 3.5844 & 2.6038 & 3.4581 \\
16 & 2.1347 & 2.9647 & 2.1072 & 2.9119 & 2.0893 & 2.8778 & 2.0457 & 2.7958 \\
18 & 1.7097 & 2.4310 & 1.6921 & 2.3954 & 1.6805 & 2.3723 & 1.6522 & 2.3163 \\
20 & 1.4035 & 2.0381 & 1.3915 & 2.0131 & 1.3837 & 1.9967 & 1.3644 & 1.9569
\end{tabular}

TABLE VI

Variation of the critical buckling load $\left(P_{\mathrm{cr}}\right)$ for different rotational restraints.

\begin{tabular}{c|c|c|c|c|c|c|c|c}
\hline \hline \multirow{2}{*}{$\mathrm{L}[\mathrm{nm}]$} & \multicolumn{2}{|c|}{$\mu=0.0 \mathrm{~nm}^{2}$} & \multicolumn{2}{c|}{$\mu=0.3 \mathrm{~nm}^{2}$} & \multicolumn{2}{c|}{$\mu=0.5 \mathrm{~nm}^{2}$} & \multicolumn{2}{c}{$\mu=1.0 \mathrm{~nm}^{2}$} \\
\cline { 2 - 9 } & $\Omega_{0}=1$ & $\Omega_{0}=1$ & $\Omega_{0}=1$ & $\Omega_{0}=1$ & $\Omega_{0}=1$ & $\Omega_{0}=1$ & $\Omega_{0}=1$ & $\Omega_{0}=1$ \\
\cline { 2 - 9 } & $\Omega_{0}=3$ & $\Omega_{0}=5$ & $\Omega_{L}=3$ & $\Omega_{L}=5$ & $\Omega_{L}=3$ & $\Omega_{L}=5$ & $\Omega_{L}=3$ & $\Omega_{L}=5$ \\
\hline 10 & 5.5995 & 5.9222 & 5.4142 & 5.7153 & 5.2974 & 5.5853 & 5.0262 & 5.2846 \\
12 & 3.9863 & 4.2443 & 3.8915 & 4.1370 & 3.8308 & 4.0684 & 3.6869 & 3.9065 \\
14 & 2.9990 & 3.2112 & 2.9450 & 3.1494 & 2.9101 & 3.1095 & 2.8263 & 3.0141 \\
16 & 2.3487 & 2.5271 & 2.3155 & 2.4886 & 2.2939 & 2.4637 & 2.2415 & 2.4034 \\
18 & 1.8965 & 2.0488 & 1.8747 & 2.0235 & 1.8605 & 2.0069 & 1.8259 & 1.9667 \\
20 & 1.5684 & 1.7002 & 1.5535 & 1.6827 & 1.5438 & 1.6712 & 1.5199 & 1.6432
\end{tabular}

In Table IV results are presented for various values of the lengths $(L=10,12,14,16,18,20 \mathrm{~nm})$. The nonlocal parameter $(\mu)$ is taken as $1.0 \mathrm{~nm}^{2}$. As can be noted, the presented results are in good agreement with those of Wang et al. [25] when the rotational restraints are taken as zero in (31). It is also seen from this table that buckling loads predicted with the rotational restraints are larger than those of classical simply supported case. This result indicates that the rotational restraints have a hardening effect.

The effect of the nonlocal parameter $(\mu)$ on the critical buckling loads of carbon nanotube is demonstrated in Tables V and VI. The results in these tables are calculated by using 100 terms of infinite series. One can observe from Tables V and VI that the critical buckling load decrease with increase in the nonlocal parameter. For the case in hand, changing the rotational restraints $\left(\Omega_{0}\right.$ and $\left.\Omega_{L}\right)$ from 1 to $5 \mathrm{nN} \mathrm{nm/(nm} \mathrm{rad)} \mathrm{re-}$ sults in an increase in the critical buckling loads, as can be noted from Tables V and VI. It can be concluded that critical buckling loads are sensitive to rotational spring parameters. The results obtained from the tables are also utilized drawing Figs. 3 and 4.

In order to compare the results for the different nonlocal parameters, the following formulation is introduced for calculating critical buckling load ratio (CBLR):

$$
\mathrm{CBLR}=P_{\mathrm{cr}}^{\mathrm{NL}} / P_{\mathrm{cr}}^{\mathrm{L}},
$$

where $P_{\mathrm{cr}}^{\mathrm{NL}}$ is the critical buckling load in nonlocal elasticity, $P_{\mathrm{cr}}^{\mathrm{L}}-$ the critical buckling load in local elasticity. In Fig. 4a-d CBLRs are plotted for various lengths and various nonlocal parameters. These figures present a comparison of the CBLRs, where the rotationally spring values are equal to 1 and $5 \mathrm{nN} \mathrm{nm/(nm} \mathrm{rad).} \mathrm{It} \mathrm{is} \mathrm{ob-}$ served that nonlocal effects increase with decrease in the length of carbon nanotube. The critical buckling loads of carbon nanotubes with hard elastic restraints are higher than those of SWCNTs with soft restraints. 


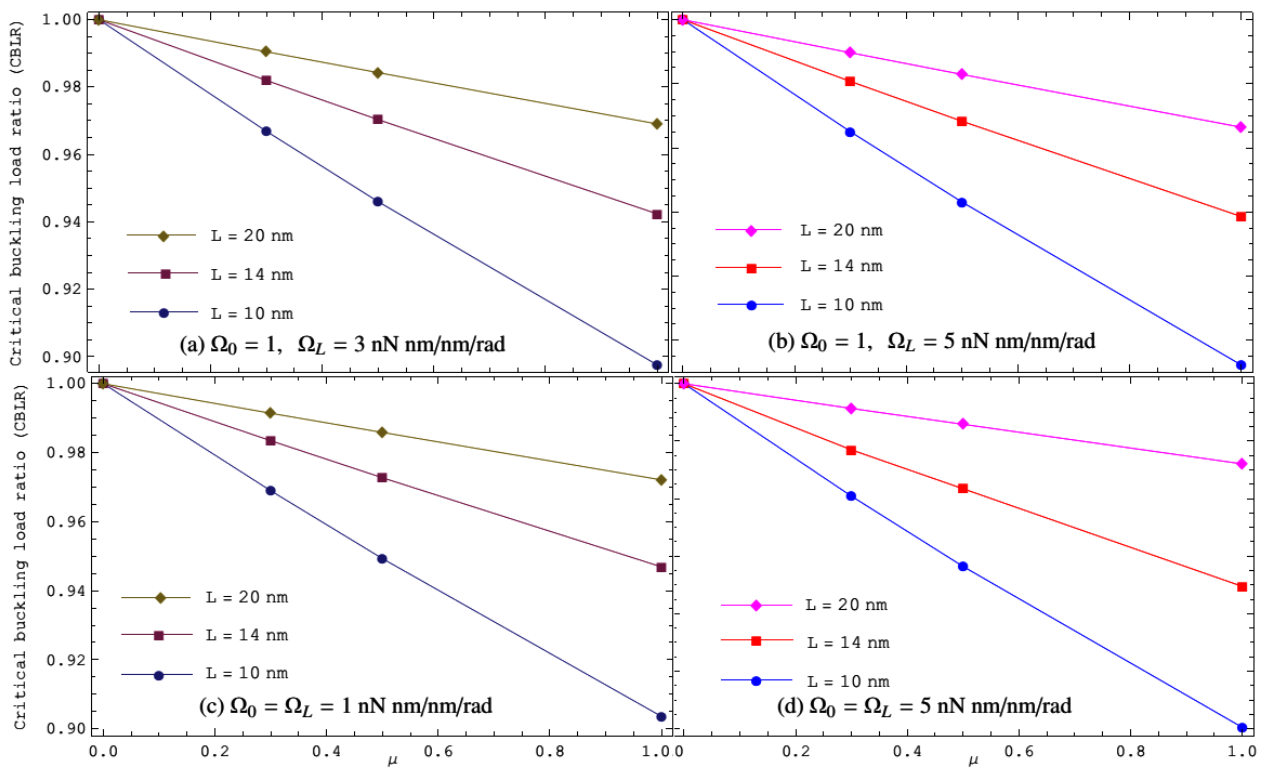

Fig. 4. Critical buckling load ratio (CBLR) for different lengths: (a) $\Omega_{0}=1, \Omega_{L}=3 \mathrm{nN} \mathrm{nm} /(\mathrm{nm} \mathrm{rad})$, (b) $\Omega_{0}=1$, $\Omega_{L}=3 \mathrm{nN} \mathrm{nm} /(\mathrm{nm} \mathrm{rad}),(\mathrm{c}) \Omega_{0}=\Omega_{L}=1 \mathrm{nN} \mathrm{nm} /(\mathrm{nm} \mathrm{rad}),(\mathrm{d}) \Omega_{0}=\Omega_{L}=5 \mathrm{nN} \mathrm{nm} /(\mathrm{nm} \mathrm{rad})$.

\section{Conclusion}

This paper investigates the stability analysis of a simply supported carbon nanotube with rotational springs. A unified analytical method is proposed, which can be used for a carbon nanotube with rigid or restrained boundary conditions. The stability determinant is constructed by a combination of Stoke's transformation and Fourier series expansion. The influence of the rotational restraints and nonlocal parameters on the critical buckling loads is examined in some numerical examples. The results indicate that small scale effects are more prominent for the carbon nanotubes with hard rotational restraints. It can also be said that the critical buckling load increases as the spring parameter increases.

\section{References}

[1] Y.Y. Zhang, C.M. Wang, W.H. Duan, Y. Xiang, Z. Zong, Nanotechnology 20, 395707 (2009).

[2] N. Yuca, N. Karatepe, F. Yakuphanoglu, Y. Hepuzer Gursel, Acta Phys. Pol. A 123, 352 (2013).

[3] O. Mermer, S. Okur, F. Sumer, C. Ozbek, S. Sayin, M. Yilmaz, Acta Phys. Pol. A 121, 240 (2012).

[4] A.C. Eringen, Int. J. Eng. Sci. 10, 1 (1972).

[5] A.C. Eringen, D.G.B. Edelen, Int. J. Eng. Sci. 10, 233 (1972).

[6] A.C. Eringen, J. Appl. Phys. 54, 4703 (1983).

[7] M. Asghari, M.H. Kahrobaiyan, M.T. Ahmadian, Int. J. Eng. Sci. 48, 1749 (2010).

[8] H.M. Ma, X.L. Gao, J.N. Reddy, J. Mech. Phys. Solids 56, 3379 (2008).
[9] W.D. Nix, H. Gao, J. Mech. Phys. Solids 46, 411 (1998).

[10] Q. Wang, K.M. Liew, Phys. Lett. A 363, 236 (2007).

[11] K. Kiani, Acta Mech. 225, 3569 (2014).

[12] K. Kiani, Curr. Appl. Phys. 14, 1116 (2014).

[13] K. Kiani, Int. J. Mech. Sci. 68, 16 (2013).

[14] K. Kiani, Physica E: Low-Dim. Syst. Nanostruct. 60, 229 (2014).

[15] J. Peddieson, G.R. Buchanan, R.P. McNitt, Int. J. Eng. Sci. 41, 305 (2003).

[16] R. Ansari, S. Sahmani, H. Rouhi, Comput. Mater. Sci. 50, 3050 (2011).

[17] T. Murmu, S.C. Pradhan, Physica E 41, 1232 (2009).

[18] D. Kumar, C. Heinrich, A.M. Waas, J. Appl. Phys. 103, 073521 (2008).

[19] M. Aydogdu, Physica E 41, 1651 (2009).

[20] R. Ansari, S. Sahmani, Int. J. Eng. Sci. 49, 1244 (2011).

[21] R. Artan, A. Tepe, Europ. J. Mech.-A/ Solids 27, 469 (2008).

[22] V. Senthilkumar, Adv. Sci. Lett. 3, 337 (2010).

[23] S.C. Pradhan, G.K. Reddy, Comput. Mater. Sci. 50, 1052 (2011).

[24] C.M. Wang, Y.Y. Zhang, S.S. Ramesh, S. Kitipornchai, J. Phys. D Appl. Phys. 39, 3904 (2006).

[25] Q. Wang, C.M. Wang, Nanotechnology 18, 075702 (2007).

[26] Q. Wang, J. Appl. Phys. 98,124301 (2005). 